1896] SUb-GRoUPS OF THE GENERAL PROJECTIVE GROUP. 221

vanish identically; whence not only

but also

$$
\xi_{0}=R_{1}-\frac{q}{2}
$$

is a root of $\varphi=0$.

Whence

$$
\begin{gathered}
R_{1}=\frac{1}{2}\left(\xi_{0}-\xi_{1}\right)=\frac{1}{2 \cdot 3^{3}}[ \\
=\frac{-\sqrt{-3}}{2.9} \sqrt{\Delta},
\end{gathered}
$$

where $\Delta=\left(x_{0}-x_{1}\right)^{2}\left(x_{1}-x_{2}\right)^{2}\left(x_{1}-x_{2}\right)^{2}$ is the discriminant of (1). Thus the suite $(\beta)$ has the character $(B)$.

YALE UNIVERSITY,

New Haven, Conn.

\title{
ON CERTAIN SUB-GROUPS OF THE GENERAL PROJECTIVE GROUP.
}

BY PROFESSOR HENRY TABER.

[Read at the January meeting of the Society, 1896.]

$$
\S 1
$$

In what follows a linear transformation homogeneous in $n$ variables as

$$
\begin{aligned}
& x_{1}{ }^{\prime}=a_{11} x_{1}+a_{12} x_{2}+\cdots+a_{1 n} x_{n}, \\
& x_{2}{ }^{\prime}=a_{21} x_{1}+a_{22} x_{2}+\cdots+a_{2 n} x_{n}, \\
& \cdots \cdots \cdots \cdots \cdots \cdots \cdots \cdots \cdots \cdots \cdots \cdots \cdots \cdots \cdots \cdots \cdots \cdots \cdots \cdots \cdots \cdots \cdots \cdots \cdots \\
& x_{n}{ }^{\prime}=a_{n 1} x_{1}+a_{n 2} x_{2}+\cdots+a_{n n} x_{n},
\end{aligned}
$$

will be denoted by the single letter $A$. If $x_{1}, x_{2}$, etc., are the Cartesian coördinates of a point in $\mathrm{n}$-fold space, the transformation $A$ is a homogeneous strain ; and the totality of transformations $A$ constitutes the group of homogeneous strains in $\mathrm{n}$-fold space. If we consider only transformations $A$ of non-zero determinant, we obtain Lie's general linear homogeneous group. The group of transformations $A$ of determinant +1 is termed by Lie the special linear homogeneous group. 
222 SUb-Groups of the GENERAL PROJECTIVE GRoUp. [April

If $B$ denotes the transformation

$$
x_{r}^{\prime \prime}=b_{r 1} x_{1}{ }^{\prime}+b_{r 2} x_{2}{ }^{\prime}+\cdots+b_{r n} x_{n}{ }^{\prime}(r=1,2, \cdots n),
$$

the transformation resulting from the successive application to $n$-fold space of $A$ and $B$ (in the order named), defined by the system of equations

$$
\begin{gathered}
x_{r}^{\prime \prime}=\sum_{1}^{n} b_{r i} a_{i 1} \cdot x_{1}+\sum_{1}^{n} b_{r i} a_{i 2} \cdot x_{2}+\cdots+\sum_{1}^{n} b_{r i} a_{i n} \cdot x_{n} \\
(r=1,2, \cdots n),
\end{gathered}
$$

will be denoted by $B A$.

If the transformation $B$ is identical with $A$, this may be indicated by writing $B=A$; and in this case the transformation $B A=B B$ may be denoted by $B^{2}$. Similarly the transformation resulting from the repetition $m-1$ times of the transformation $B$ (that is, the transformation resulting from $m$ successive applications of $B$ ) may be denoted by $B^{m}$, which may be termed the $m^{\text {th }}$ power of $B$.

If for $r, s=1,2, \cdots n$,

$$
b_{r r}=1, b_{r s}=0(r \neq s),
$$

$B$ is the identical transformation. The letter $I$ will be employed to denote the identical transformation. We have $I A=A I=A$. We may denote the transformation for which

$$
b_{r r}=\rho, b_{r s}=0(r \neq s),
$$

by $\rho I$; and the transformation $\rho I$. $A$, defined by the equations $x_{r}^{\prime}=\rho a_{r 1} x_{1}+\rho a_{r 2} x_{2}+\cdots+\rho a_{r n} x_{n}(r=1,2, \cdots n)$ may be denoted simply by $\rho A$. In particular, the transformation defined by the equations

$x_{r}{ }^{\prime}=-a_{r 1} x_{1}-a_{r 2} x_{2}-\cdots-a_{r n} x_{n}(r=1,2, \ldots n)$ will be denoted by $-A$.

The transformation $B$ is infinitesimal if

$$
b_{r r}=1+c_{r r} \delta t, \quad b_{r s}=c_{r s} \delta t \quad(r \neq s),
$$

where $c_{r r}$ and $c_{r s}$ are constants, and $\delta t$ is an infinitesimal quantity.

Following Sylvester, I shall say that the nullity of $B$ is $m$ if the minor determinants of order $n-m+1$ of the matrix 
1896] SUB-GROUPS OF THE GENERAL PROJECTIVE GROUP. 223

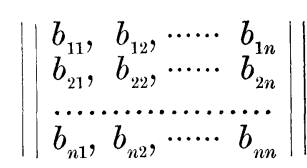

are all zero, but not all the minors of order $n-m$.

If we denote by $A_{\lambda}$ the transformation

$$
\begin{aligned}
& x_{1}^{\prime}=\left(a_{11}-\lambda\right) x_{1}+a_{12} x_{2}+\cdots a_{1 n} x_{n}, \\
& x_{2}^{\prime}=a_{21} x_{1}+\left(a_{22}-\lambda\right) x_{2}+\cdots+a_{2 n} x_{n}, \\
& \cdots \cdots \cdots \cdots \cdots \cdots \cdots \cdots \cdots \cdots \cdots \cdots \cdots \cdots \cdots \cdots \cdots \cdots \cdots \cdots \cdots \cdots \cdots \cdots \cdots \cdots \cdots \cdots \\
& x_{n}^{\prime}==a_{n 1} x_{1}+a_{n 2} x_{2}+\cdots+\left(a_{n n}-\lambda\right) x_{n},
\end{aligned}
$$

where $\lambda$ is a root of multiplicity $\mu$ of the characteristic equation of $A$,

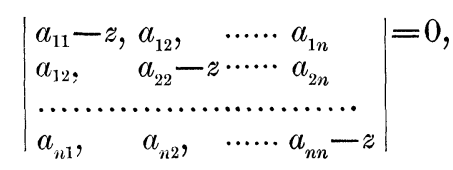

the nullity of $A_{\lambda}$ is at least one, and cannot exceed $\mu$. If the nullity of $A_{\lambda}$ is equal to $\mu$, the nullity of $A_{\lambda}^{2}, A_{\lambda}^{3}$, etc., is also $\mu$. On the other hand, if the nullity of $A_{\lambda}$ is less than $\mu$, the nullity of $A_{\lambda}^{2}$ is greater by one, at least, than the nullity of $A_{\lambda}$; and the nullity of successive powers of $A_{\lambda}$ increases until a power of exponent $\nu$ is attained whose nullity is $\mu$. The nullity of the $(\nu+1)^{\text {th }}$ and higher powers of $A_{\lambda}$ is then also $\mu$. If we denote by

$$
\mu_{1}, \mu_{2}, \cdots \mu_{\nu-1}, \mu_{\nu}=\mu,
$$

respectively, the nullities of

we have

$$
A_{\lambda}, A_{\lambda}^{2}, \cdots A_{\lambda,}^{\nu-1} A_{\lambda}^{\nu}
$$

$$
\mu_{1} \geqq \mu_{2}-\mu_{1} \geqq \mu_{3}-\mu_{2} \geqq \ldots \geqq \mu_{\nu}-\mu_{\nu-1} \geqq 1 .
$$

I shall term the numbers $\mu_{1}, \mu_{2} \cdots \mu_{\nu}$, the numbers belonging to the root $\lambda$ of the characteristic equation $A$. We can always find a transformation $A$ which shall have any complex number $\lambda$ as a root of its characteristic equation, and any set of numbers, subject only to the above conditions, as the numbers belonging to that root. To subject the numbers belonging to any root of the characteristic equation of $A$ to any condition other than those given above, is of course, equivalent to imposing certain conditions upon the coefficients of $A$. 
The real linear homogeneous group $G$, for $n$ variables, and the group of of real projective transformations in $(n-1)$ fold space are connected as follows. If the variables $x_{1}, x_{2}$, etc., are regarded as the homogeneous coördinates of a point in $(n-1)$-fold space, the real linear transformation $A$ homogeneous in the $x$ 's determines a corresponding real projective transformation $\mathfrak{A}$ in $(n-1)$-fold space. In the determination of this projective transformation, we are concerned only with the ratios of the coefficients of $A$; consequently, the transformations $A$ and $\rho A$ of group $G$ (where $\rho$ is any real number) correspond to the same projective transformation $\mathfrak{A}$. The identical transformation $I$ and the transformation $\rho I$ of group $G$ correspond to the same projective transformation $\mathbb{E}$, the identical transformation of group (A. Let $B$ be a transformation of group $G$ corresponding to the real projective transformation $\mathbf{B B}$ : if $A=B^{m}$, then $\mathfrak{A}=\mathfrak{b}^{m}$; and, if $\mathfrak{A}=\mathbf{b}^{m}$, then $\rho A=B^{m}$ for some real quantity $\rho$. Consequently, if either $A$ or $\rho A$ is the $m^{t h}$ power of a transformation of group $G$, the corresponding projective transformation $\mathcal{A}$ of group $\mathbb{G}$ is the $m^{\text {th }}$ power of some transformation of this group, and not otherwise. Further, if $B$ is infinitesimal, the corresponding projective transformation $\mathbb{1 3}$ differs infinitely little from the identical transformation of group $\mathbb{C}$. Therefore, if either $A$ or $\rho A$ can be generated by the repetition of an infinitesimal transformation of group $G$, the corresponding projective transformation of group can be generated by the repetition of an infinitesimal transformation of this group and not otherwise.

If $\bar{G}$ denotes the group of real linear transformations of determinant +1 homogeneous in $n$ variables (the real special linear homogeneous group), every transformation $\bar{A}$ of group $\bar{G}$ determines a (corresponding) transformation of group $\mathbb{6}$. If $n$ the number of variables is odd, there is a one to one correspondence between the transformations of group $\bar{G}$ and the transformations of the real projective group. For in this case (that is, if $n$ is odd), if $A$ is a real linear transformation homogeneous in the variables corresponding to the real projective transformation $\mathfrak{A}$, the trans-

formation $\bar{A}=\frac{1}{\mathrm{a}} A$, where a denotes the real $n^{\text {th }}$ root of

the determinant of $A$, is a transformation of group $\bar{G}$ and the only transformation of this group the ratios of whose coefficients are the same as the ratios of the coefficients of 
$A$. Consequently, when $n$ is odd, if $\bar{A}$ is the $m^{\text {th }}$ power of a transformation of group $G$, the corresponding real projective transformation $\mathfrak{A}$ is the $m^{\text {th }}$ power of a transformation of group of and conversely; also if $\bar{A}$ can be generated by the repetition of an infinitesimal transformation of group $G$, $\mathbb{A}$ can be generated by the repetition of an infinitesimal transformation of group (F, and conversely. On the other hand, if $n$ is even, and if the determinant of $A$ (the real linear transformation homogeneous in the variables, determining the real projective transformation $\mathfrak{A}$ ) is positive, there are corresponding to $\mathfrak{A}$ two transformations of group $\bar{G}$ obtained by dividing each coefficient of $A$ by either the positive or by the negative real $n^{\text {th }}$ root of the determinant of $A$; but if the determinant of $A$ is negative there is no transformation of group $\bar{G}$ corresponding to $\mathfrak{A}$.

\section{$\S 2 . *$}

In the Comptes Rendus, volume 94, Sylvester showed that every transformation $A$ of non-zero determinant is the $m^{\text {th }}$ power for any positive integer $m$ of some transformation $B$. That is, if $A$ is any transformation of non-zero determinant, a transformation $B$ can always be found, for any positive integer $m$, such that $A=B^{m}$. In his paper, Sylvester also gave a formula expressing the coefficients of $B$ as algebraic functions of the coefficients of $A$. From a subsequent theorem of Sylvester's, $\dagger$ it follows that, if $m$ is sufficiently great, we may take $B$ to differ as little as we please from the identical transformation.

For real linear transformations homogeneous in $n$ variables we have the following theorems.

Theorem I. If the transformation $A$ is real and of non-zero determinant (or of determinant +1 ), a real transformation $B$ of non-zero determinant (or of determinant +1 ) can always be found of which $A$ is the $(2 m+1)^{\text {th }}$ power for any odd number $2 m+1$. But in order that $A$ may be any even power of a real transformation $B$ of non-zero determinant (or of determinant +1 ), it is necessary and sufficient that all the numbers belonging to each negative root of the characteristic equation of $A$ shall be even. Therefore, in order that the real transformation $A$ of non-zero determinant (or of determinant +1 ) may be any even power of a real transformation $B$ of non-zero determinant (or of determinant

* Throughout this section, by transformation is meant a linear transformation homogeneous in $n$ variables.

† Johns Hopkins University Circulars. 
+1 ) it is necessary and sufficient that $A$ shall be the second power of a real transformation of non-zero determinant (or of determinant +1$)$.

Theorem II. If $A$ is the second power of a real transformation of non-zero determinant (or of determinant +1 ), a real transformation $B$ of non-zero determinant (or of determinant+1) can always be found which shall be as nearly as we please equal to the identical transformation, and such that for some positive integer $m$ sufficiently great $B^{m}$ shall be equal to $A$. Consequently, any transformation that is the second power of a real transformation of nonzero determinant can be generated by the repetition of a real infinitesimal transformation; and, in particular, a transformation that $i s$ the second power of a real transformation of determinant +1 can be generated by the repetition of an infinitesimal transformation of determinant +1 .

Theorem III. If the real transformation $A$ of non-zero determinant (or of determinant +1 ) is not the second power of a real transformation of non-zero determinant (or of determinant +1 ), nevertheless, a real transformation $B$ of non-zero determinant (or of determinant +1 ) can be found whose coefficients are algebraic functions of a parameter $t$ such that $A$ shall be the limit towards which the transformation $B^{2}$ approaches as $t$ approaches zero, provided the multiplicity of each negative root of the characteristic equation of $A$ is even.

If $\lambda$ is any root of the characteristic equation of the real transformation $B$, and if, for $r, s=1,2, \cdots n$,

$$
\left|1-b_{r r}\right|<\varepsilon,\left|b_{r s}\right|<\varepsilon(r \neq s),
$$

where $\varepsilon$ is any positive quantity, then provided $\varepsilon$ is sufciently small, the absolute value of $1-\lambda$ may be made as small as we please ; and consequently no root of the characteristic equation of $B$ will be negative or equal to zero. Therefore, by Theorem I, if $\varepsilon$ is sufficiently small, $B$ is the second power of a real transformation $B_{1}$ of non-zero determinant; and, in particular, if $B$ is of determinant +1 , $B_{1}$ may be so chosen that its determinant shall be equal to +1 . For any positive integer $m, B^{m}$ is equivalent to the second power of $B_{1}{ }^{m}$; that is, $B^{m}=\left(B_{1}^{2}\right)^{m}=\left(B_{1}{ }^{m}\right)^{2}$. Whence we have the following theorem.

Theorem IV. The repetition of a real infinitesimal transformation results in a transformation which is the second power of a real transformation. In particular, the repetition of a real infinitesimal transformation of determinant +1 results in a transforma- 
tion which is the second power of a real transformation of determinant +1 .

From Theorem III follows:

Theorem V. By the repetition of a real infinitesimal transformation, we may approach as near as we please to any real transformation $A$ of non-zero determinant, provided the multiplicity of each negative root of the characteristic equation of $A$ is even. In particular if these conditions are satisfied, and if the determinant of $A$ is +1 , we may approach as near as we please to $A$ by the repetition of a real infinitesimal transformation of determinant +1 .

If the transformation $A$ is not the second power of a real transformation, it may be that $-A$ is the second power of a real transformation. The roots of the characteristic equation of $-A$ are the negatives of the roots of the characteristic equation of $A$. Whence follows from Theorem I :

Theorem VI. In order that the real transformation $-A$ of non-zero determinant shall be the second power of a real transformation, it is necessary and sufficient that the numbers belonging to each positive root of the characteristic equation of $A$ shall all be even.

\section{$\S 3$.}

In the Bulletin for July, 1894, I pointed out that not every orthogonal substitution can be generated by the repetition of an infinitesimal orthogonal substitution, and if $G$ is the group of proper orthogonal substitutions I showed that

Theorem VII. If we designate a transformation of the group $G$ as of the first or second kind according as it is or is not the second power of a transformation of the group, then every transformation of the first kind is the $m^{\text {th }}$ power of a transformation of the group for any positive integer $m$, and can be generated by the repetition of an infinitesimal transformation of the group. By definition no transformation of the second kind is any even power of a transformation of the group. Nor can any transformation of the second kind be generated by the repetition of an infinitesimal transformation of the group. But every transformation of the second kind is the $(2 m+1)^{t h}$ power of a transformation of the group for any odd exponent $2 m+1$.

In a note at the end of this paper, I mentioned that Theorem VII was true of the group of linear transformations homogeneous in $n$ variables that transform into itself a bilinear form (of non-zero determinant understood) with co- 
grediant variables. This was afterwards demonstrated for an alternate bilinear form in a paper published in volume 46 of the Mathematische Annalen.

In a paper presented to the American Academy of Arts and Sciences, April, 1895, I have shown that Theorem VII holds for the class of linear transformations homogeneous in $n$ variables that transform a bilinear form (of non-zero determinant) with contragrediant variables into itself. The transformations of this class that are commutative form a a group for which also Theorem VII holds.

My attention has recently been called to a note in the Bulletin for November, 1893,* stating that Professors Engel and Study have found that not every transformation of the special linear homogeneous group for two variables can be generated by the repetition of an infinitesimal transformation of that group. There is nothing in the note to show that the real special linear homogeneous group is referred to. $\dagger$

I learn from Prof. H. B. Newson that he has found that not every transformation of the real projective group can be generated by the repetition of an infinitesimal transformation of that group. This discovery suggested the extension of Theorem VII to other sub-groups of the projective group. It follows at once from Newson's discovery that not every transformation of the real linear homogeneous group can be generated by the repetition of an infinitesimal transformation of this group ; and further that not every transformation of the real special linear homogeneous group for an odd number of variables can be generated by the repetition of an infinitesimal transformation of this group. For could every transformation of either of these groups be generated by the repetition of an infinitesimal transformation of that group, then, as pointed out in $\$ 1$, every transformation of the real projective group could be generated by the repetition of an infinitesimal transformation of this group, which is not the case.

It follows from Theorems I, II and IV, that Theorem VII is true for the real linear homogeneous group for $n$ variables,

\footnotetext{
* Page 66.

†Theorem VII holds for the special linear homogeneous group in two variables; and the transformation $x^{\prime}=-x+y, y^{\prime}=-y$ is not the second power of a transformation of this group, and therefore cannot be generated by the repetition of an infinitesimal transformation of this group. From the note it would appear that the identical transformation $x^{\prime}=x, y^{\prime}=y$, and the involutional transformation $x^{\prime}=-x, y^{\prime}=-y$ can not be generated by the repetition of an infinitesimal transformation of the group, which is erroneous.
} 
and also for the group of real linear transformations of dederminant +1 homogeneous in $n$ variables (the real special linear homogeneous group); and, from what is stated in the latter part of $\S 1$, that Theorem VII holds for the real projective group.

In volume 26 , page $36 t$ of the troceedings of the London Mathematical Society, I have sh own hat in order that an orthogonal substitution may be of the first kind (that is, in order that a transformation of the orthogonal group may be generated by the repetition of an infinitesimal orthogonal transformation), it is necessary and sufficient that either -1 shall not be a root of the characteristic equation of the substitution or that the numbers belonging to the root -1 shall all be even. In a paper read at the Summer Meeting, 1895, of the American Mathematical Society (to appear in the Quarterly Journal of Mathematics) I showed that a transformation of the group of linear transformations homogeneous in the variables that transform an alternate bilinear form with cogrediant variables into itself will be of the first kind under the same conditions.*

By Theorem I, in order that a transformation of the real linear homogeneous group (or of the real special linear homogeneous group) shall be of the first kind it is necessary and sufficient that the numbers belonging to each negative root of the characteristic equation of the transformation shall all be even.

Therefore, by $\$ 1$, in order that a transformation of the real projective group shall be of the first kind. it is necessary and sufficient, either that the numbers belonging to each positive root of the characteristic equation of the transformation shall all be even, or else that the numbers belonging to each negative root of the characteristic equation shall all be even; provided there are both positive and negative real roots. Otherwise no condition is necessary.

In the article in the Bulletin on orthogonal substitution above mentioned I showed that by the repetition of an infinitesimal orthogonal substitution we can approximate as nearly as please to any proper orthogonal substitution of the second kind. In the above mentioned paper in the Mathematische Annalen I showed that by the repetition of an

* In the paper I also showed that every orthogonal transformation of this group is of the first kind. Also, if denotes a bilinear form of nonzero determinant with cogrediant variables, and eff $^{\prime}$ its conjugate ; and if

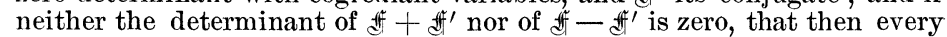
linear transformation homogeneous in the variables that transforms into itself is the $m^{\text {th }}$ power of such a transformation for any exponent $m$. 
infinitesimal transformation of the group of linear transformations homogeneous in the variables that transform an alternate bilinear form into itself we can approximate as nearly as we please to any transformation of the second kind belonging to this group.

From Theorem $\mathrm{V}$ it follows that by the repetition of an infinitesimal transformation of the real linear homogeneous group (or of the real special Jinear homogeneous group) we may approximate as nearly as we please to any transformation of the second kind belonging to this group, provided the multiplicity of each negative root of the characteristic equation of this transformation is even.

Therefore by the repetition of an infinitesimal transformation of the real projective group we may approximate as nearly as we please to any transformation of the second kind belonging to this group, provided that either the multiplicity of each positive root of the characteristic equation of this transformation is even or else that the multiplicity of each negative root of the characteristic equation is even.

Worcester, Mass.

\section{POSTSCRIP'T.}

The general real linear group and the special real linear group. The totality of linear transformations

$$
y_{r}^{\prime}=\mathrm{a}_{r 1} y_{1}+\mathrm{a}_{r^{2}} y_{2}+\cdots+\mathrm{a}_{r, n-1} y_{n-1}+\mathrm{a}_{r_{n},}(r=1,2, \cdots n-1)
$$

for which the determinant $\sum \pm \mathrm{a}_{11} \mathrm{a}_{22} \cdots \mathrm{a}_{n-1, n-1}$ is not zero form a group termed by Lie the general linear group. The sub-group of transformations for which the determinant $\Sigma \pm \mathrm{a}_{11} \mathrm{a}_{22} \cdots \mathrm{a}_{n-1, n-1}$ is +1 , Lie terms the special linear group. These two groups are obviously identical with the two groups of linear transformations $A$ that are respectively of non-zero determinant and of determinant +1 , homogeneous in $n$ variables and defined by the equations

$$
\begin{aligned}
& x_{r}^{\prime}=a_{r 1} x_{1}+a_{r 2} x_{2}+\cdots+a_{r n} x_{n}(r=1,2, \cdots n-1), \\
& x_{n}^{\prime}=x_{n},
\end{aligned}
$$

For the general real linear group, and for the special real linear group, Theorem VII holds.* The conditions neces-

* Every transformation of the general linear group is the $m^{t h}$ power of a transformation of this group for any exponent $m$, and can be generated by the repetition of an infinitesimal transformation of this group, if we consider transformation of this group both imaginary and real. The same is true of the special linear group. 
sary and sufficient that a transformation $A$ of the general real linear group (or of the special real linear group) may be of the first kind are that the numbers belonging to each negative root of the characteristic equation of $A$ shall all be even. By the repetition of an infinitesimal transformation of the real linear group we may approximate as nearly as we please to any transformation $A$ of the second kind belonging to this group, provided the multiplicity of each negative root of the characteristic equation of is $A$ even, but not otherwise. In particular, if these conditions are satisfied (but not otherwise), and if $A$ is of determinant +1 , we may approximate as nearly as we please to $A$ by the repetition of an infinitesimal transformation of the special real linear group.

Special linear homogeneous group. Let $A$ be any linear transformation (real or imaginary) of determinant +1 homogeneous in $n$ variables, that is, let $A$ be any transformation of the special linear homogeneous group. Let

$$
\lambda^{\prime}=r_{1} e^{\theta_{1} V=1} \quad, \quad \lambda^{\prime \prime}=r_{2} e^{\theta_{2} V-1} \quad, \text { etc. }
$$

(where $\theta_{1}, \theta_{2}$, etc., are the smallest positive arguments of $\lambda^{\prime}, \lambda^{\prime \prime}$, etc.,) be the roots of the characteristic equation of $A$ of respectively multiplicity $m^{\prime}, m^{\prime \prime}$, etc. Since the determinant of $A$ is +1 , we have

$$
\begin{gathered}
r_{1}^{m^{\prime}} r_{2}^{m^{\prime \prime}} \cdots \cdots=1, \\
m^{\prime} \theta_{1}+m^{\prime \prime} \theta_{2}+\cdots \cdots=2 k \pi,
\end{gathered}
$$

for some positive integer $k$.

If $k=0$, the transformation $A$ can be generated by the repetition of an infinitesimal transformation of the special linear homogeneous group. The conditions in general necessary and sufficient in order that $A$ may be generated by the repetition of an infinitesimal transformation of this group are somewhat complicated; they depend upon $k$, and upon the numbers belonging to the roots $\lambda$ of the characteristic equation of $A$. The conditions may be exhibited as follows.

Let the numbers belonging to $\lambda^{\prime}$ be

$$
m_{1}{ }^{\prime}, m_{2}{ }^{\prime}, \cdots \cdots \cdots m_{\nu^{\prime}-1}, m^{\prime}{ }_{\nu^{\prime}}=m^{\prime} ;
$$

let the numbers belonging to $\lambda^{\prime \prime}$ be

$$
m_{1}{ }^{\prime \prime}, m_{2}{ }^{\prime \prime}, \cdots \cdots \cdots \cdot m_{\nu^{\prime \prime}-1}, m^{\prime \prime}{ }_{\nu^{\prime \prime}}=m^{\prime \prime} ;
$$


232 sub-groups of the general PROJECtive group. [April

etc., etc. Form a diagram corresponding to $\lambda^{\prime}$ by arranging $m^{\prime}$ dots in rows and columns so that there shall be $\nu^{\prime}$ rows respectively of

$$
m_{1}^{\prime}, m_{2}^{\prime}-m_{1}^{\prime}, \ldots \ldots m_{\nu^{\prime}}^{\prime}-m_{\nu^{\prime}-1}^{\prime}
$$

dots. The number of columns will then be $m_{1}^{\prime}$. In one column there will be $\nu^{\prime}$ dots. Let the number of dots in these $m_{1}^{\prime}$ columns be respectively

$$
p_{1}{ }^{\prime}, p_{2}{ }^{\prime}, \cdots \cdots p_{m_{1}^{\prime}}^{\prime} *{ }^{*}
$$

* If the numbers belonging to $\lambda^{\prime}$ are $5,9,13,16$, the diagram corresponding to $\lambda^{\prime}$ is

and $\quad p_{1}{ }^{\prime}=p_{2}{ }^{\prime}=p_{3}{ }^{\prime}=4, \quad p_{4}{ }^{\prime}=3, \quad p_{5}{ }^{\prime}=1$,

$$
\begin{aligned}
& * * * * * \\
& * * * * \\
& * * * * \\
& * * *
\end{aligned}
$$

Next form a diagram corresponding to $\lambda^{\prime \prime}$ by arranging $m^{\prime \prime}$ dots in rows and columns so that there shall be $\nu^{\prime \prime}$ rows respectively of

$$
m_{1}^{\prime \prime}, m_{2}^{\prime \prime}-m_{1}^{\prime \prime}, \ldots \ldots m^{\prime \prime}{ }_{\nu^{\prime \prime}}-m^{\prime \prime}{ }_{\nu^{\prime \prime}-1}
$$

dots; and let the number of dots in these $m_{1}{ }^{\prime \prime}$ columns be respectively

$$
p_{1}^{\prime \prime}, p_{2}^{\prime \prime}, \cdots \cdots \cdot p^{\prime \prime}{ }_{m_{1}}
$$

Proceed in the same way to form diagrams corresponding to the other roots of the characteristic equation of $A$.

Let now $\delta$ be the greatest common divisor of the $p$ 's. Then if $k$ contain $\delta, A$ can be generated by the repetition of an infinitesimal transformation of the special linear homogeneous group and not otherwise. In particular, if $m^{\prime}, m^{\prime \prime}$, etc., have no common factor, $A$ can be generated by the repetition of an infinitesimal transformation of the special linear homogeneous group.

If the transformation $A$ can be generated by the repetition of an infinitesimal transformation of the special linear homogeneous group, $A$ is the $m^{\text {th }}$ power of a transformation of this group for any exponent $m$.

If $\delta_{1}$ is the greatest common divisor of $k$ and $\delta$, then $A$ is the $m^{\text {th }}$ power of a transformation of the special linear homogeneous group for any exponent $m$ that contains no factor of $\delta$ except a factor of $\delta_{1}$; but for no other exponent is $A$ 
the $m^{\text {th }}$ power of a transformation of the special linear homogeneous group. In particular if $m$ is relatively prime to $n$, the number of variables, $A$ is the $m^{\text {th }}$ power of a transformation of the special linear homogeneous group.

If $A$ cannot be generated by the repetition of an infinitesimal transformation of the special linear homogeneous group, the $m^{\text {th }}$ power of $A$ can be generated thus, provided $m$ contains $\delta / \delta_{1}$ (the quotient of $\delta$ by the greatest common divisor of $\delta$ and $k$ ), but not otherwise.

March 4, 1896.

\section{NOTES.}

A Regular meeting of the American Mathematical Society was held in New York on Saturday afternoon, February 29, at three o'clock, the President Dr. HILl, in the chair. There were eleven members present. On the recommendation of the Council, Mr. Harry Brown Evans, University of Pennsylvania, Philadelphia, Pa., was elected to membership. The following papers were read :

(1) Professor M. I. Pupin: "On the integral of the differential equation

$$
a \frac{d^{2} y}{d t^{2}}+b \frac{d y}{d t}=c \frac{d^{2} y}{d x^{2}}
$$

under certain boundary conditions occurring in the theory of long electrical waves."

(2) Professor E. H. MOORE: "A two-fold generalization of Fermat's theorem."

In the absence of Professor Moore his paper was read by the Secretary.

Among the courses for the summer semester at Berlin are the following-Professor Fuchs: Introduction to the theory of differential equations; Introduction to the theory of functions;-Professor Frobenius: Theory of numbers(second course); Theory of determinants;-Professor Schwarz: Analytical geometry; Applications of the theory of the elliptic functions; Hyper-geometric series; Mathematical colloquium;-Professor Knoblauch, Theory of the elliptic functions; Theory of curved surfaces; Theory of space curves; -Professor Hettner: Introduction to the theory of infinite series, products, and continued fractions;-ProfessorHensel: Concerning the transcendentalism of $e$ and $\pi$; Higher alge- 